International Journal of Modern Physics A

(C) World Scientific Publishing Company

\title{
ARE THERE STRANGELETS IN COSMIC RAYS?
}

\author{
M. RYBCZYŃSKI and Z. WŁODARCZYK \\ Institute of Physics, Świętokrzyska Academy, ul. Świętokrzyska 15, 25-406 Kielce, Poland \\ G. WILK \\ The Andrzej Soltan Institute for Nuclear Studies, Zd-P8, ul. Hoża 69; 00-681 Warsaw, Poland
}

Received (Day Month Year)

Revised (Day Month Year)

\begin{abstract}
Assuming that cosmic rays entering the Earth's atmosphere contain a small admixture of nuggets of strange quark matter in form of strangelets one can explain a number of apparently "strange" effects observed in different cosmic rays experiments. We shall demonstrate here that the mass spectrum of such strangelets filles the "nuclear desert" gap existing between the heaviest elements observed in Universe and the next "nuclearlike objects" represented by neutron and strange stars.
\end{abstract}

Keywords: Strange Quark Matter; strangelets.

Cosmic ray experiments of different kinds abound in results which are rather unexpected when considered with the experience rooted in the accelerator physics only 12. The most spectacular observations are: anomalous cosmic ray bursts from Cygnus $X$-3, extraordinary high luminosity gamma ray burst from the supernova remnant $N_{4} 9$ in the Large Magellanic Cloud, or the so called Centauro events, which are characterised by anomalous composition of secondary particles with no neutral pions present. As demonstrated in detail in 23 substantial part of such events can be explained by assuming that cosmic rays entering the Earth's atmosphere contain a small admixture of nuggets of strange quark matter (SQM) in form of strangelets, i.e., of nuggets with $200<A<10^{6}$ (below lower limit stangelets become unstable while above upper limit they contain also electrons and then their size is limited only by the unstability due to gravitational collaps which happens at $A \sim 10^{57}$ ). Such SQM is probably continuosulsy produced in some neutron stars (or in quark stars). For example, Chandra observatory has discovered an object, which probably could be a strange star 4 . One should mention here that recently one event with small ratio $Z / A$ have been found experimentally with AMS detector ${ }^{5}$ (albeit with $A$ very small, estimated to be $A \simeq 17.5$, i.e., it would be a metastable strangelet).

In this presentation we would like to discuss one more feature of strangelets, namely the fact that they seem to fill the gap, or the nuclear desert, existing between the heaviest elements observed in Universe and the next nuclear-like objects 
represented by neutron stars (or possibly by strange and quark stars) 46. They do by following the empirical (not so well known) $A^{-7.5}$ power law dependence found long time ago in 7 when analysing the abundance of the elements in the Universe, see Fig. [1

So far this $A^{-7.5}$ dependence (and, in general, the reason for such power law to show up) is, to our knowledge, unexplained. We would like to propose here very simple reasoning leading to such behaviour. Let us consider an object (nucleus or strangelet) carrying mass number $A_{i}$, which absorbes neutrons with probability $W=\Phi \sigma=A_{i} v \sigma$. In time $t$ it travels distance $L=v t$. If absorption of neutrons proceeds with cross section $\sigma$ and if density of neutrons is $n$, then the increment of mass of such object in a unit time is $\partial A_{i}=n A_{i} L \sigma \partial t / t$. For the $i$-th such object one has then that

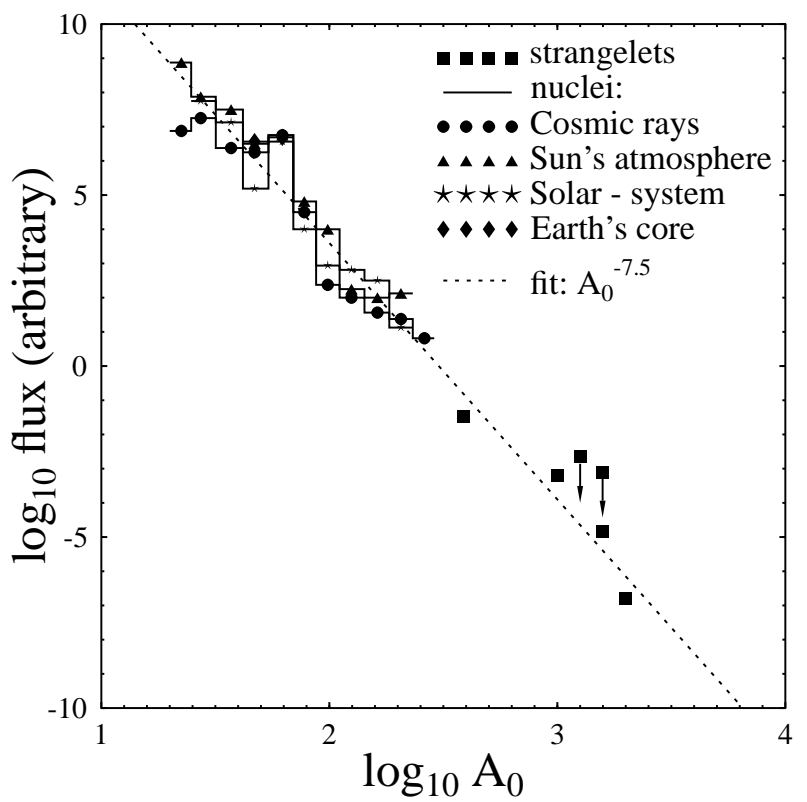

Fig. 1. Comparison of the estimated mass spectrum $N\left(A_{0}\right)$ of strangelets with the known abundances of elements in the Universe 7 . Consecutive steps in histogram denote the following nuclei (or groups of nuclei): $\mathrm{Ne},(\mathrm{Mg}, \mathrm{Si}), \mathrm{S},(\mathrm{K}, \mathrm{Ca}), \mathrm{Fe},(\mathrm{Cu}, \mathrm{Zn}),(\mathrm{Kr}, \mathrm{Sr}, \mathrm{Zr}),(\mathrm{Te}, \mathrm{Xe}, \mathrm{Ba})$, (Rare earths), $(\mathrm{Os}, \mathrm{Ir}, \mathrm{Pt}, \mathrm{Pb})$. The flux of strangelets used here has been obtained from attempt to accomodate together all possible signals of strangelets 223 .

$$
\frac{\partial A_{i}}{A_{i}}=\alpha \cdot \frac{\partial t}{t} \quad \Longrightarrow \quad A_{i}(t)=m\left(\frac{t}{t_{i}}\right)^{\alpha}
$$

where $\alpha=n L \sigma$. The initial condition used here is that the $i$-th object occurs in time $t_{i}$ with mass $A_{i}\left(t_{i}\right)=m$. Probability of forming an object of mass $A_{i}(t)<A$ 
is then

$$
P\left(A_{i}(t)<A\right)=p\left(t_{i}>\frac{m^{1 / \alpha} \cdot t}{A^{1 / \alpha}}\right) .
$$

Assuming now uniform distribution of the occurence of such objects in the system, i.e., that the probability of adding such object in the unit time interval to the system is $P\left(t_{i}\right)=1 / t$, one gets that

$$
P\left(t_{i}>\frac{m^{1 / \alpha} \cdot t}{A^{1 / \alpha}}\right)=1-P\left(t_{i} \leq \frac{m^{1 / \alpha} \cdot t}{A^{1 / \alpha}}\right)=1-\frac{m^{1 / \alpha}}{A^{1 / \alpha}} .
$$

This, together with eq. (2), results in the following expression for the probability of forming an object of mass $A$ :

$$
P(A)=\frac{\partial P\left(A_{i}(t)<A\right)}{\partial A}=\frac{m^{1 / \alpha}}{1+1 / \alpha} \cdot A^{-(1+1 / \alpha)} .
$$

It means then that in this way we have obtained the following time-independent power-like distribution of abundances of elements of mass number $A$ :

$$
P(A) \propto A^{-\gamma} \quad \text { with } \quad \gamma=1+\frac{1}{\alpha}=1+\frac{1}{n L \sigma} .
$$

In our case $\gamma=7.5$ corresponds to $\alpha=n L \sigma=0.154$ and this, for the typical value of the cross section entering here equal to $\sigma=30 \mathrm{mb}$, gives the thickness of the layer in which our objets are to be produced equal to $n L=5.1 \cdot 10^{24} \mathrm{~cm}^{-2}=8.5 \mathrm{~g} / \mathrm{cm}^{2}$. Assuming now for the typical density of neutrons value of $n \sim 10^{20} \mathrm{~cm}^{-3}=1.6 \cdot 10^{-4}$ $\mathrm{g} / \mathrm{cm}^{3}$ and assuming that the so-called $r$-process nucleosynthesis conditions are met 8 one gets that this can be achieved with a rather thin layer of thickness equal to $L=0.5 \mathrm{~km}$.

To summarize: we have reminded that SQM can still be interesting topic of research, in particular in what concerns strangelets and their properties. In particular we have provided a viable explanation of the specific $A$-dependence of their abundance in terms of the usual nucler processes.

\section{References}

1. G. Wilk and Z. Włodarczyk, Phys. Rev. D50, 2318 (1994); Acta Phys. Polon. A27, 2649 (1996); Nucl. Phys. B (Proc. Suppl.) A75, 191 (1999).

2. M. Rybczyński, Z. Włodarczyk and G. Wilk, Nucl. Phys. B (Proc. Suppl.) 97, 85 (2001) and Acta Phys. Polon. B33, 277 (2001).

3. G. Wilk and Z. Włodarczyk, J. Phys. G22, L105 (1996); Heavy Ion Phys. 4, 395 (1996).

4. F. Walter, Astrop. J. 549, 433 (2001); S. Murray et al., Astrop. J. 568, 226 (2002); J.J. Drake et al., Astrop. J. 572, 226 (2002); P.O. Slane, D.J. Helfand and S. Murray, Astrop. J. 571, L45 (2002).

5. M. Pohl, these proceedings; V. Choutko (AMS Coll.), 28 ICRC (2003) OG1, 1765.

6. L. Zdunik, P. Haensel and E. Gourgoulhon, Astron. and Astrophys. 372, 535 (2001); L. Zdunik, Astron. and Astrophys. 359, 311 (2001); F. Weber, astro-ph/0407155 to be published in Prog. Part. Nucl. Phys. (2004).

7. G.B. Zhdanov, Usp. Fiz. Nauk 111, 109 (1973) [Sov. Phys. Uspekhi 16, 642 (1974)].

8. B.S. Meyer, Ann. Rev. Astron. Astrophys. 32, 153 (1994). 
International Journal of Modern Physics A

(C) World Scientific Publishing Company

\title{
ARE THERE STRANGELETS IN COSMIC RAYS?
}

\author{
M. RYBCZYŃSKI and Z. WŁODARCZYK \\ Institute of Physics, Świętokrzyska Academy, ul. Świętokrzyska 15, 25-406 Kielce, Poland \\ G. WILK \\ The Andrzej Soltan Institute for Nuclear Studies, Zd-P8, ul. Hoża 69; 00-681 Warsaw, Poland
}

Received (Day Month Year)

Revised (Day Month Year)

\begin{abstract}
Assuming that cosmic rays entering the Earth's atmosphere contain a small admixture of nuggets of strange quark matter in form of strangelets one can explain a number of apparently "strange" effects observed in different cosmic rays experiments. We shall demonstrate here that the mass spectrum of such strangelets filles the "nuclear desert" gap existing between the heaviest elements observed in Universe and the next "nuclearlike objects" represented by neutron and strange stars.
\end{abstract}

Keywords: Strange Quark Matter; strangelets.

Cosmic ray experiments of different kinds abound in results which are rather unexpected when considered with the experience rooted in the accelerator physics only ${ }^{1,2}$. The most spectacular observations are: anomalous cosmic ray bursts from Cygnus $X$-3, extraordinary high luminosity gamma ray burst from the supernova remnant $N_{4} 9$ in the Large Magellanic Cloud, or the so called Centauro events, which are characterised by anomalous composition of secondary particles with no neutral pions present. As demonstrated in detail in ${ }^{2,3}$ substantial part of such events can be explained by assuming that cosmic rays entering the Earth's atmosphere contain a small admixture of nuggets of strange quark matter (SQM) in form of strangelets, i.e., of nuggets with $200<A<10^{6}$ (below lower limit stangelets become unstable while above upper limit they contain also electrons and then their size is limited only by the unstability due to gravitational collaps which happens at $A \sim 10^{57}$ ). Such SQM is probably continuosulsy produced in some neutron stars (or in quark stars). For example, Chandra observatory has discovered an object, which probably could be a strange star ${ }^{4}$. One should mention here that recently one event with small ratio $Z / A$ have been found experimentally with AMS detector ${ }^{5}$ (albeit with $A$ very small, estimated to be $A \simeq 17.5$, i.e., it would be a metastable strangelet).

In this presentation we would like to discuss one more feature of strangelets, namely the fact that they seem to fill the gap, or the nuclear desert, existing between the heaviest elements observed in Universe and the next nuclear-like objects 
represented by neutron stars (or possibly by strange and quark stars) ${ }^{4,6}$. They do by following the empirical (not so well known) $A^{-7.5}$ power law dependence found long time ago in ${ }^{7}$ when analysing the abundance of the elements in the Universe, see Fig. 1.

So far this $A^{-7.5}$ dependence (and, in general, the reason for such power law to show up) is, to our knowledge, unexplained. We would like to propose here very simple reasoning leading to such behaviour. Let us consider an object (nucleus or strangelet) carrying mass number $A_{i}$, which absorbes neutrons with probability $W=\Phi \sigma=A_{i} v \sigma$. In time $t$ it travels distance $L=v t$. If absorption of neutrons proceeds with cross section $\sigma$ and if density of neutrons is $n$, then the increment of mass of such object in a unit time is $\partial A_{i}=n A_{i} L \sigma \partial t / t$. For the $i$-th such object one has then that

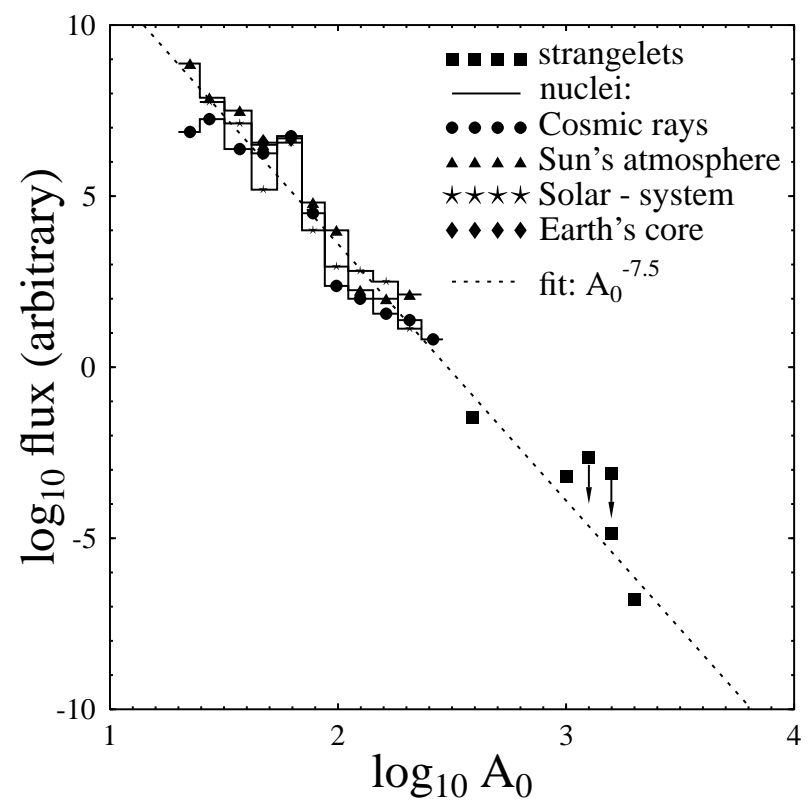

Fig. 1. Comparison of the estimated mass spectrum $N\left(A_{0}\right)$ of strangelets with the known abundances of elements in the Universe ${ }^{7}$. Consecutive steps in histogram denote the following nuclei (or groups of nuclei): $\mathrm{Ne},(\mathrm{Mg}, \mathrm{Si}), \mathrm{S},(\mathrm{K}, \mathrm{Ca}), \mathrm{Fe},(\mathrm{Cu}, \mathrm{Zn}),(\mathrm{Kr}, \mathrm{Sr}, \mathrm{Zr}$ ), (Te,Xe,Ba), (Rare earths), $(\mathrm{Os}, \mathrm{Ir}, \mathrm{Pt}, \mathrm{Pb})$. The flux of strangelets used here has been obtained from attempt to accomodate together all possible signals of strangelets ${ }^{2,3}$.

$$
\frac{\partial A_{i}}{A_{i}}=\alpha \cdot \frac{\partial t}{t} \quad \Longrightarrow \quad A_{i}(t)=m\left(\frac{t}{t_{i}}\right)^{\alpha}
$$

where $\alpha=n L \sigma$. The initial condition used here is that the $i$-th object occurs in time $t_{i}$ with mass $A_{i}\left(t_{i}\right)=m$. Probability of forming an object of mass $A_{i}(t)<A$ 
is then

$$
P\left(A_{i}(t)<A\right)=p\left(t_{i}>\frac{m^{1 / \alpha} \cdot t}{A^{1 / \alpha}}\right) .
$$

Assuming now uniform distribution of the occurence of such objects in the system, i.e., that the probability of adding such object in the unit time interval to the system is $P\left(t_{i}\right)=1 / t$, one gets that

$$
P\left(t_{i}>\frac{m^{1 / \alpha} \cdot t}{A^{1 / \alpha}}\right)=1-P\left(t_{i} \leq \frac{m^{1 / \alpha} \cdot t}{A^{1 / \alpha}}\right)=1-\frac{m^{1 / \alpha}}{A^{1 / \alpha}} .
$$

This, together with eq. (2), results in the following expression for the probability of forming an object of mass $A$ :

$$
P(A)=\frac{\partial P\left(A_{i}(t)<A\right)}{\partial A}=\frac{m^{1 / \alpha}}{1+1 / \alpha} \cdot A^{-(1+1 / \alpha)} .
$$

It means then that in this way we have obtained the following time-independent power-like distribution of abundances of elements of mass number $A$ :

$$
P(A) \propto A^{-\gamma} \quad \text { with } \quad \gamma=1+\frac{1}{\alpha}=1+\frac{1}{n L \sigma} .
$$

In our case $\gamma=7.5$ corresponds to $\alpha=n L \sigma=0.154$ and this, for the typical value of the cross section entering here equal to $\sigma=30 \mathrm{mb}$, gives the thickness of the layer in which our objets are to be produced equal to $n L=5.1 \cdot 10^{24} \mathrm{~cm}^{-2}=8.5 \mathrm{~g} / \mathrm{cm}^{2}$. Assuming now for the typical density of neutrons value of $n \sim 10^{20} \mathrm{~cm}^{-3}=1.6 \cdot 10^{-4}$ $\mathrm{g} / \mathrm{cm}^{3}$ and assuming that the so-called $r$-process nucleosynthesis conditions are met 8 one gets that this can be achieved with a rather thin layer of thickness equal to $L=0.5 \mathrm{~km}$.

To summarize: we have reminded that SQM can still be interesting topic of research, in particular in what concerns strangelets and their properties. In particular we have provided a viable explanation of the specific $A$-dependence of their abundance in terms of the usual nucler processes.

\section{References}

1. G. Wilk and Z. Włodarczyk, Phys. Rev. D50, 2318 (1994); Acta Phys. Polon. A27, 2649 (1996); Nucl. Phys. B (Proc. Suppl.) A75, 191 (1999).

2. M. Rybczyński, Z. Włodarczyk and G. Wilk, Nucl. Phys. B (Proc. Suppl.) 97, 85 (2001) and Acta Phys. Polon. B33, 277 (2001).

3. G. Wilk and Z. Włodarczyk, J. Phys. G22, L105 (1996); Heavy Ion Phys. 4, 395 (1996).

4. F. Walter, Astrop. J. 549, 433 (2001); S. Murray et al., Astrop. J. 568, 226 (2002); J.J. Drake et al., Astrop. J. 572, 226 (2002); P.O. Slane, D.J. Helfand and S. Murray, Astrop. J. 571, L45 (2002).

5. M. Pohl, these proceedings; V. Choutko (AMS Coll.), 28 ICRC (2003) OG1, 1765.

6. L. Zdunik, P. Haensel and E. Gourgoulhon, Astron. and Astrophys. 372, 535 (2001); L. Zdunik, Astron. and Astrophys. 359, 311 (2001); F. Weber, astro-ph/0407155, to be published in Prog. Part. Nucl. Phys. (2004).

7. G.B. Zhdanov, Usp. Fiz. Nauk 111, 109 (1973) [Sov. Phys. Uspekhi 16, 642 (1974)].

8. B.S. Meyer, Ann. Rev. Astron. Astrophys. 32, 153 (1994). 